\title{
Following the ethanol TRAIL to prostate
} cancer destruction

umour necrosis factor-related
apoptosis-inducing ligand
(TRAIL) - a molecule that is naturally produced as part of the body's immune response-has shown great potential as an anticancer therapy in laboratory-based studies and preclinical trials. Although TRAIL has little effect on healthy cells, this protein induces apoptosis in a broad range of cancer cells, including prostate carcinomas. However, some cell lines are more sensitive to TRAIL than others; for example, androgen-resistant PC3 cells are moderately sensitive, whereas androgen-dependent LNCaP cells are refractory to TRAIL-induced apoptosis. Encouragingly, resistance can often be overcome by combining TRAIL with other chemotherapeutics.

In a recent study, Plante and colleagues have shown that ethanol-an agent that is known to selectively destroy hepatoma tumour cells-can also enhance the proapoptopic effects of TRAIL on prostate cancers. "The use of TRAIL for inducing apoptosis in various cancer cell lines, including prostate cancer cells, has gained much attention," says Uma Wesley, who led the study. "However, some tumour cells exhibit resistance or decreased sensitivity to TRAIL-induced cytotoxicity. The main goal of our study was to establish whether ethanol enhances TRAIL's apoptotic potential, and to understand the molecular mechanisms associated with its effects."

Plante et al. exposed two of the most commonly studied prostate cancer cell lines-PC3 and LNCaP-to TRAIL, ethanol, and a combination of these agents for $48 \mathrm{~h}$ and studied cell morphology, proliferation, apoptosis (using annexin- $\mathrm{V}$ binding and TUNEL assays), reactive oxygen species (ROS) production, anchorage-independent growth, and $3 \mathrm{D}$-matrigel invasion. The investigators clearly demonstrated that the cytotoxic effects of TRAIL were more pronounced in the presence of ethanol.

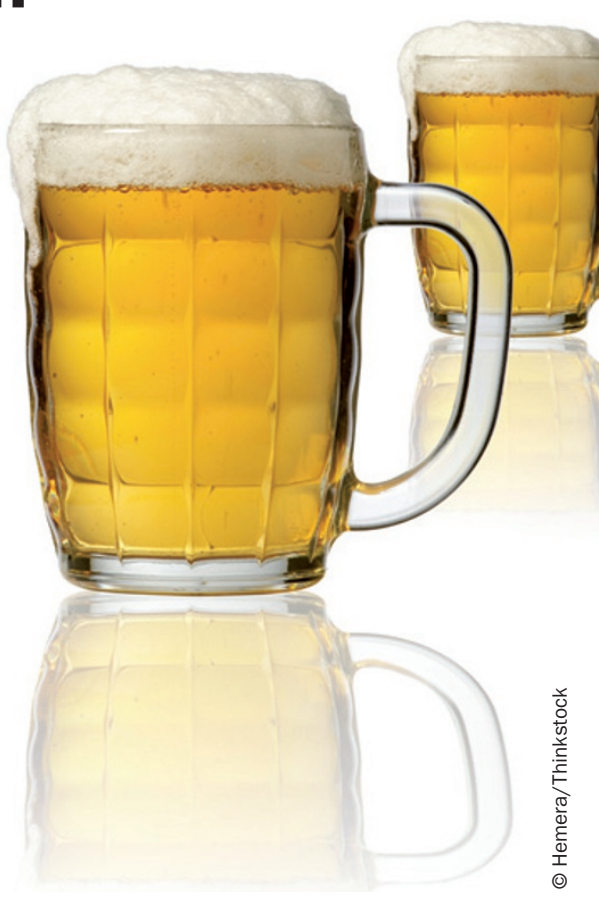

Cells treated with both agents demonstrated rounded morphology and signs of detachment, as well as significant reductions in viability. Levels of apoptosis were notably greater in cells treated with combination therapy, reaching 58\% in PC3 cells and $40 \%$ in LNCaP cells (compared with $28 \%$ and $14 \%$, respectively, with TRAIL alone). This effect was exerted via caspase activation and poly (ADP) ribose polymerase (PARP) cleavage, as well as downregulation of the antiapoptopic protein Bcl-2 and Phos-AKT, upregulation of the apoptosis inducer cyclin-dependent kinase (cdk) inhibitor 1A (also known as $\mathrm{p} 21$ ), and increased production of ROS. Inhibition of colony formation was also more pronounced in cells treated with combined ethanol and TRAIL (compared with either single treatment) and cells were less able to migrate and invade matrigel-coated filters.

Importantly, the research team also investigated the in vivo effects of combined ethanol and TRAIL treatment. Male nude $\mathrm{BALB} / \mathrm{c}$ mice were injected with PC3

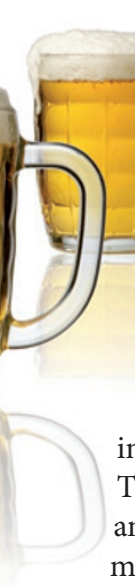
removed. In accordance with in vitro data, levels of intratumoural apoptosis were significantly higher in animals exposed to combined therapy rather than single-agent treatment. Although these initial findings are encouraging, Wesley stresses that further preclinical studies investigating dose, timing, and drug toxicityparticularly over longer durations-are required to determine the therapeutic value of ethanol in combination therapy.

Mark Plante-who conceived the original idea for the study and collaborated closely with Wesley-is a firm advocate of injection therapy for prostatic conditions. "Much published clinical evidence demonstrates that transurethral intraprostatic injection of $98 \%$ ethanol is effective for the treatment of symptomatic BPH," says Plante, the Director of Urologic Research at the University of Vermont College of Medicine. "However, although injection therapy is a longstanding concept, it remains in its infancy for widespread clinical application. There is no doubt that targeted injection of these agents will be possible in the future, but we are years away from applicability."

Melanie Clyne

Original article Plante, M. K. et al. Ethanol promotes cytotoxic effects of tumor necrosis factor-related apoptosisinducing ligand through induction of reactive oxygen species in prostate cancer cells. Prostate Cancer Prostatic Dis. doi:10.1038/pcan.2012.37 